\title{
K- Mean and Euclidian Cluster Analysis for Salt Tolerance Rice Genotypes under Alkaline Soil Condition
}

\author{
Ashutosh Kashyap*, Vijay Kumar Yadav, Poonam Singh, P. K. Singh and Shweta \\ Department of Genetics and Plant Breeding, Chandra Shekhar Azad University of Agriculture \\ \& Technology, Kanpur-(U.P.) India \\ *Corresponding author
}

\section{A B S T R A C T}

\begin{tabular}{l} 
K e y w o r d s \\
Rice, genotypes, K- \\
clustering, \\
$\begin{array}{l}\text { Euclidian } \\
\text { clustering, Salt } \\
\text { tolerance, Rice, } \\
\text { Sodicity }\end{array}$ \\
Article Info \\
$\begin{array}{l}\text { Accepted: } \\
\text { 04 October } 2020 \\
\text { Available Online: } \\
10 \text { November } 2020\end{array}$ \\
\hline
\end{tabular}

\section{Introduction}

Rice is the most important staple food crop of the world. It is the principal food of half of the world's human population inhabiting the humid tropics and subtropics. World population is increasing rapidly by every passing year and there will be a need to produce $87 \%$ more of what we are producing today especially food crops such as rice, wheat, soy and maize by 2050 (Kromdijk and Long, 2016). Sodicity is one of the major soil constraints to crop production and is expected to increase due to global climate changes and as a consequence of many irrigation practices.
An experiment was conducted to examine K- Mean Cluster and Euclidian Cluster analysis on 78 genotypes including seven standards (checks) varieties viz., CSR36, CSR10, CST71, CSR27, Usar Dhan 3 for salinity and alkalinity tolerant, while Sambha Sub1 as for general stress, and PUSA 44 as salt stress sensitive were grown in Augmented Randomized Block Design to selecting salt tolerance and breaking the yield barrier under alkaline soil condition. All genotypes were grouped into nine clusters by both k-Means Clustering, and Euclidian revealed the genotypes of heterogeneous origin were frequently present in same cluster. Low conformity was observed in placing of genotypes in both have -2016-IR-18-10 placed as highest second yielder followed by CSA -2016, CARI dhan 10, Usar Dhan 3 possessed $4^{\text {th }}, 16^{\text {th }}$ and $25^{\text {th }}$ rank. These genotypes were considered with high yielder and more stable across the environments. 
algorithm is simple, efficient, high and easy to handle data has been applied to many areas. However, K-means algorithm is very sensitive to initialize, the better center. This study was undertaken to determine the genetic diversity in salt tolerant rice genotypes for the maximum utilization of the genetic resources and proper selection of donor parents with using both $\mathrm{K}$ Cluster Mean and Euclidian cluster analysis.

\section{Materials and Methods}

The experiment was conducted during year 2017 and 2018, at Crop Research Farm, Nawabganj and Seed Multiplication Farm Bojha, Chandra Sheker Azad University of Agriculture and Technology, Kanpur (U.P.) India on 71 rice genotypes and seven checks varieties viz., CSR36, CSR10, CST7-1, CSR27, Sambha Sub1, Usar Dhan 3 for sodicity resistant and, and PUSA44 as salt stress sensitive in Augmented Randomized Block Design with replications of check under three environments taking into consideration of soil types and days of sowing. The details of the environments are given below: Environments:E-1: Environment I, Year 2017, high stress, $\mathrm{pH}$ 9.8, Ec 1.43 $\mathrm{dsm}^{-1}$, Seed Multiplication Farm, Bojha; E-2: Environment II, Year 2018, high stress, pH 9.8, Ec1.41 $\mathrm{dsm}^{-1}$, Seed Multiplication Farm, Bojha; E-3: Environment III, Year 2018, Normal stress, pH 8.8, Ec0.96dsm ${ }^{-1}$ CRF, Nawabganj.

Five plants in all genotype and checks were selected at random from each replication for recording of observations on characters of these genotype were used for recording all the below mentioned characters. The average of observations recorded on these five plants was considered for statistical analysis. Plant morphological characters of each genotype were recorded by selecting single or group of plants depending on all characters at different stages of crop growth. Days to 50\% flowering Plant height $(\mathrm{cm})$, Total no of tillers plant ${ }^{-1}$, Number of panicle bearing tillers plant ${ }^{-1}$, Panicle Length $(\mathrm{cm})$ Filled grain panicle ${ }^{-1}$, Spikelet fertility percentage, 1000- grain weight (g), Stress score at reproductive stage and Grain yield plant ${ }^{-1}$.

The genotypes were grouped into clusters based on Mahalanobis's D2 statistics and canonical variate analysis and $\mathrm{K}$ cluster mean analysis by K-means method (Hartigan and Wang, 1979; Lloyd, 1957; Mac Queen, 1967 on the basis of average distance of k-means and the accessions in each cluster were then analyzed for basic statistics.

\section{Results and Discussion}

The aim of clustering is to provide measures and criteria that are used for determining whether two objects are similar or dissimilar. In present study, two types of clustering techniques k-Means Clustering and Hierarchical Euclidian clustering were used to characterization of genotypes based on genetic divergence for selection of suitable and diverse genotypes (Manju et al., 2014). These procedures characterize genetic divergence using the criterion of similarity or dissimilarity based on the aggregate effects of a number of yield contributing important characters.

The k-means clustering algorithm is a centroid based approach using cluster distortion to decide when sufficient progress has been made but also can be restricted to a certain number of iterations (Hartigan and Wong 1979). Convergence of the algorithm is based on the change in distance of the mean cluster distance metric. This distance metric is often the squared Euclidean distance or squared normal distance between an observation and the centroid (Fig. 1-3). 
Table.1 Mean performance of 78 genotypes for 10 characters in Oryza sativa

\begin{tabular}{|c|c|c|c|c|c|c|c|c|c|c|c|}
\hline S. No. & Character & $\begin{array}{c}\text { Days to } \\
50 \% \\
\text { flowering }\end{array}$ & $\begin{array}{c}\text { Plant } \\
\text { height } \\
(\mathrm{cm})\end{array}$ & Tillers/Plant & $\begin{array}{l}\text { Productive } \\
\text { Tillers/plant }\end{array}$ & $\begin{array}{l}\text { Panicle } \\
\text { Length } \\
(\mathrm{cm})\end{array}$ & $\begin{array}{c}\text { Filled } \\
\text { grains/panicle }\end{array}$ & $\begin{array}{l}\text { Spikelet } \\
\text { Fertility } \\
(\%)\end{array}$ & $\begin{array}{c}\text { Test } \\
\text { Weight }\end{array}$ & $\begin{array}{l}\text { Stress score } \\
\text { at } \\
\text { reproductive } \\
\text { stage }\end{array}$ & $\begin{array}{c}\text { Grain } \\
\text { Yield } \\
\text { g/plant }\end{array}$ \\
\hline 1 & CARI Dhan 10 & 85.33 & 82.90 & 8.53 & 5.67 & 19.06 & 149.33 & 76.28 & 19.00 & 2.00 & 31.46 \\
\hline 2 & CARI Dhan 11 & 85.33 & 153.83 & 15.83 & 12.43 & 23.39 & 134.67 & 82.40 & 20.73 & 1.00 & 36.08 \\
\hline 3 & CARI Dhan 6 & 104.00 & 105.63 & 11.10 & 9.17 & 20.92 & 130.67 & 83.92 & 23.40 & 2.00 & 27.45 \\
\hline 4 & CR 2851-S-1-6-B-B-4 & 121.33 & 76.67 & 10.93 & 8.93 & 21.83 & 105.67 & 74.83 & 19.67 & 7.00 & 12.35 \\
\hline 5 & CR 2851-S-B-1-2B-1 & 96.67 & 92.07 & 15.03 & 11.27 & 19.55 & 122.67 & 75.10 & 23.47 & 3.00 & 27.00 \\
\hline 6 & CR 2851-S-B-1-B-B-1 & 127.33 & 104.53 & 14.33 & 10.67 & 21.07 & 96.00 & 71.54 & 22.68 & 7.00 & 8.58 \\
\hline 7 & CR 3437-1*-S-200-83-1 & 105.00 & 90.17 & 11.50 & 8.43 & 23.33 & 138.67 & 77.91 & 17.20 & 5.00 & 22.28 \\
\hline 8 & CR 3878-245-2-4-1 & 95.33 & 102.00 & 10.70 & 9.27 & 22.09 & 123.67 & 75.44 & 20.82 & 3.00 & 28.48 \\
\hline 9 & CR 3880-10-1-9-2-2-1 & 101.00 & 79.37 & 11.57 & 6.37 & 18.81 & 114.00 & 73.88 & 19.80 & 3.00 & 24.16 \\
\hline 10 & CR 3881-4-1-3-7-2-3 & 114.00 & 112.10 & 13.50 & 10.03 & 21.14 & 121.00 & 73.37 & 19.97 & 7.00 & 12.88 \\
\hline 11 & CR 3883-3-1-5-2-1-2 & 94.00 & 116.20 & 7.70 & 5.60 & 20.22 & 97.67 & 72.50 & 23.47 & 3.00 & 23.92 \\
\hline 12 & CR 3884-244-8-5-11-1-1 & 99.33 & 67.40 & 11.33 & 7.67 & 22.11 & 104.00 & 78.89 & 22.57 & 7.00 & 9.47 \\
\hline 13 & CR 3887-15-1-2-1 & 91.67 & 116.20 & 11.07 & 9.53 & 19.25 & 113.67 & 71.53 & 17.90 & 2.00 & 31.41 \\
\hline 14 & CR 3890-35-1-3-4 & 104.33 & 113.07 & 14.67 & 10.20 & 24.99 & 132.67 & 80.89 & 22.70 & 2.00 & 35.92 \\
\hline 15 & CR 3904-162-1-5-1 & 125.33 & 97.07 & 7.33 & 5.67 & 22.75 & 94.67 & 69.56 & 22.33 & 7.00 & 10.87 \\
\hline 16 & CR3881-M-3-1-5-1-1-1 & 94.33 & 98.10 & 13.33 & 10.20 & 22.52 & 115.00 & 71.22 & 20.28 & 3.00 & 30.96 \\
\hline 17 & CR3882-7-1-6-2-2-1 & 92.67 & 77.47 & 10.27 & 8.07 & 24.57 & 126.67 & 78.47 & 19.01 & 3.00 & 24.57 \\
\hline 18 & CR3884-244-8-5-6-1-1 & 100.67 & 96.68 & 15.10 & 10.27 & 22.13 & 111.67 & 77.62 & 18.82 & 7.00 & 14.58 \\
\hline 19 & CR3903-161-1-3-2 & 119.33 & 104.67 & 10.33 & 8.07 & 18.93 & 114.67 & 76.93 & 22.67 & 6.33 & 19.95 \\
\hline 20 & CSA 2016-IR18-6 & 91.00 & 113.87 & 13.17 & 11.93 & 20.87 & 149.00 & 87.20 & 22.83 & 1.00 & 36.72 \\
\hline 21 & CSAR 1604 & 92.33 & 78.23 & 10.33 & 7.60 & 19.82 & 122.67 & 73.28 & 24.60 & 3.00 & 27.81 \\
\hline 22 & CSAR 1610 & 91.33 & 90.20 & 11.77 & 9.00 & 20.43 & 112.67 & 68.92 & 23.73 & 2.00 & 29.88 \\
\hline 23 & CSAR 1628 & 105.33 & 104.51 & 9.93 & 7.90 & 19.67 & 78.67 & 67.50 & 24.03 & 5.00 & 18.94 \\
\hline 24 & CSAR1620 & 96.67 & 83.23 & 13.50 & 11.87 & 20.99 & 151.67 & 84.42 & 23.30 & 2.00 & 36.77 \\
\hline 25 & CSR 2016-IR18-1 & 96.67 & 75.53 & 12.00 & 9.33 & 21.61 & 119.67 & 76.69 & 21.54 & 7.00 & 16.41 \\
\hline 26 & CSR 2016-IR18-10 & 94.33 & 121.23 & 13.80 & 11.00 & 19.48 & 123.67 & 79.85 & 22.62 & 1.00 & 37.10 \\
\hline 27 & CSR 2016-IR18-11 & 108.33 & 103.07 & 13.37 & 10.17 & 19.75 & 120.67 & 76.64 & 20.83 & 5.00 & 18.28 \\
\hline 28 & CSR 2016-IR18-12 & 95.33 & 103.20 & 12.03 & 9.03 & 26.03 & 103.67 & 72.35 & 24.37 & 3.00 & 31.87 \\
\hline 29 & CSR 2016-IR18-14 & 114.00 & 77.28 & 12.87 & 10.93 & 19.90 & 108.33 & 77.49 & 18.97 & 6.67 & 12.34 \\
\hline 30 & CSR 2016-IR18-15 & 105.00 & 88.70 & 13.23 & 8.80 & 24.37 & 105.67 & 71.78 & 21.30 & 3.00 & 25.74 \\
\hline 31 & CSR 2016-IR18-16 & 105.67 & 84.03 & 12.43 & 7.67 & 24.97 & 64.67 & 65.84 & 22.48 & 3.00 & 19.41 \\
\hline 32 & CSR 2016-IR18-17 & 100.00 & 102.80 & 14.60 & 12.67 & 20.60 & 120.33 & 79.20 & 24.61 & 3.00 & 27.18 \\
\hline 33 & CSR 2016-IR18-18 & 99.67 & 103.83 & 10.77 & 8.60 & 24.58 & 115.00 & 77.35 & 24.37 & 3.00 & 30.28 \\
\hline 34 & CSR 2016-IR18-2 & 116.33 & 94.00 & 11.17 & 8.17 & 24.70 & 121.67 & 78.02 & 21.57 & 4.33 & 23.75 \\
\hline 35 & CSR 2016-IR18-3 & 107.33 & 85.03 & 11.93 & 9.93 & 20.91 & 128.00 & 85.47 & 24.65 & 3.00 & 26.07 \\
\hline 36 & CSR 2016-IR18-5 & 110.33 & 90.48 & 15.00 & 12.67 & 25.60 & 79.33 & 70.20 & 20.83 & 3.00 & 23.17 \\
\hline 37 & CSR 2016-IR18-7 & 95.33 & 101.23 & 13.07 & 9.37 & 21.04 & 99.00 & 83.14 & 22.43 & 5.00 & 20.38 \\
\hline 38 & CSR 2016-IR18-8 & 96.00 & 102.60 & 11.50 & 9.33 & 24.13 & 124.00 & 75.81 & 22.50 & 2.00 & 30.78 \\
\hline 39 & CSR 2016-IR18-9 & 81.33 & 75.57 & 13.90 & 11.00 & 18.70 & 98.33 & 74.85 & 22.13 & 5.00 & 12.61 \\
\hline 40 & CSR RIL-01-IR165 & 101.67 & 106.40 & 10.77 & 7.93 & 22.23 & 121.00 & 79.11 & 21.88 & 2.00 & 32.93 \\
\hline 41 & CSR-2748-197 & 84.00 & 102.07 & 17.87 & 13.93 & 22.75 & 120.33 & 77.19 & 20.77 & 2.00 & 30.88 \\
\hline 42 & CSR-2748-4441-193 & 77.00 & 125.23 & 9.43 & 7.10 & 23.41 & 118.67 & 82.54 & 23.50 & 1.00 & 35.22 \\
\hline 43 & CSR-2748-4441-195 & 95.00 & 82.93 & 14.60 & 11.97 & 22.46 & 119.67 & 75.47 & 18.73 & 2.00 & 27.26 \\
\hline 44 & CSRC(S)47-7-B-B-1-1 & 87.00 & 137.87 & 11.17 & 7.57 & 20.56 & 102.67 & 77.49 & 19.03 & 3.00 & 30.90 \\
\hline 45 & CSR-C27SM-117 & 92.00 & 96.27 & 12.93 & 10.73 & 18.25 & 105.00 & 72.18 & 24.90 & 2.00 & 33.95 \\
\hline 46 & $\begin{array}{l}\text { IR 83421-6-B-3-1-1 CR } \\
3364-S-2\end{array}$ & 96.33 & 91.20 & 14.77 & 11.20 & 20.69 & 119.00 & 76.51 & 23.53 & 3.00 & 21.30 \\
\hline 47 & IR10206-29-2-1-1 & 105.67 & 105.80 & 10.77 & 7.50 & 19.88 & 120.00 & 79.06 & 18.07 & 7.00 & 16.90 \\
\hline 48 & IR52280-117-1-1-3 & 93.33 & 128.30 & 12.87 & 9.40 & 25.31 & 116.00 & 72.27 & 22.55 & 2.00 & 34.62 \\
\hline 49 & $\begin{array}{l}\text { IR84649-81-4-1-3B- } \\
\text { CR3397-S-B-4 }\end{array}$ & 96.00 & 95.27 & 7.23 & 5.50 & 19.80 & 108.33 & 81.69 & 22.69 & 3.00 & 28.51 \\
\hline 50 & KR 15010 & 78.00 & 74.97 & 10.50 & 8.77 & 24.94 & 129.33 & 79.04 & 17.73 & 7.00 & 17.92 \\
\hline 51 & KR 15016 & 82.00 & 87.17 & 11.80 & 9.23 & 17.48 & 136.00 & 79.81 & 18.77 & 5.00 & 20.18 \\
\hline 52 & KR15006 & 78.67 & 90.83 & 15.77 & 12.77 & 25.92 & 134.00 & 83.16 & 25.23 & 3.00 & 29.33 \\
\hline 53 & $\mathrm{KS}-12$ & 84.00 & 70.03 & 14.53 & 11.50 & 21.79 & 113.00 & 84.86 & 25.24 & 1.00 & 38.12 \\
\hline 54 & NDRK 11-20 & 86.00 & 94.50 & 16.13 & 12.03 & 21.04 & 123.67 & 76.29 & 23.37 & 2.00 & 29.08 \\
\hline 55 & NDRK 11-21 & 86.00 & 92.77 & 12.17 & 9.60 & 21.44 & 99.00 & 75.46 & 27.23 & 3.00 & 29.36 \\
\hline
\end{tabular}




\begin{tabular}{|c|c|c|c|c|c|c|c|c|c|c|c|}
\hline 56 & NDRK 11-22 & 94.33 & 100.23 & 12.93 & 10.27 & 22.07 & 103.00 & 67.10 & 24.67 & 3.00 & 20.91 \\
\hline 57 & NDRK 11-24 & 84.67 & 91.17 & 10.97 & 8.47 & 23.65 & 124.67 & 76.73 & 25.43 & 2.00 & 31.66 \\
\hline 58 & PAU 3835-12-1-1-1 & 103.33 & 86.94 & 16.00 & 11.67 & 20.74 & 121.33 & 82.45 & 22.00 & 5.00 & 22.03 \\
\hline 59 & PAU 3835-36-6-3-3-4 & 84.67 & 77.53 & 10.43 & 8.43 & 23.80 & 109.67 & 69.57 & 21.70 & 5.00 & 18.40 \\
\hline 60 & PAU 4254-14-1-2-2-2-4-1 & 115.33 & 101.40 & 12.43 & 9.83 & 21.26 & 114.33 & 72.53 & 21.07 & 5.00 & 16.38 \\
\hline 61 & PAU 5563-23-1-1 & 108.00 & 75.53 & 10.27 & 8.33 & 20.46 & 104.33 & 71.37 & 18.50 & 5.00 & 26.08 \\
\hline 62 & PAU 7114-3480-1-1-1-0 & 85.67 & 103.70 & 12.17 & 10.00 & 22.07 & 140.00 & 80.51 & 20.90 & 2.00 & 28.05 \\
\hline 63 & RAU $1397-14$ & 104.67 & 85.23 & 15.33 & 12.60 & 19.60 & 111.00 & 79.52 & 20.40 & 2.00 & 32.86 \\
\hline 64 & RP 5440-302-100-7-6-3-2 & 110.00 & 66.40 & 13.53 & 9.27 & 19.98 & 113.00 & 79.09 & 18.07 & 5.00 & 16.25 \\
\hline 65 & RP 5687-420-111-5-4-2-1 & 115.00 & 100.87 & 14.13 & 8.40 & 22.63 & 140.33 & 81.22 & 19.70 & 7.00 & 18.23 \\
\hline 66 & RP 5694-36-9-5-1-1 & 109.00 & 107.07 & 16.80 & 13.53 & 23.13 & 113.00 & 76.76 & 20.85 & 5.00 & 17.80 \\
\hline 67 & RP-320-4-3-2-1 & 115.33 & 67.27 & 11.53 & 7.67 & 24.29 & 156.33 & 75.90 & 18.80 & 7.00 & 10.85 \\
\hline 68 & RP-5683-101-85-30-2-3-1 & 96.00 & 71.53 & 10.27 & 8.70 & 18.21 & 113.00 & 78.15 & 23.00 & 3.00 & 19.86 \\
\hline 69 & Sambha Sub1 & 120.00 & 87.97 & 15.43 & 12.83 & 18.71 & 128.00 & 77.64 & 22.58 & 7.00 & 19.59 \\
\hline 70 & TR 09027 & 87.00 & 63.87 & 13.33 & 11.10 & 19.60 & 87.33 & 72.42 & 18.63 & 5.33 & 13.05 \\
\hline 71 & TR 09030 & 95.67 & 87.53 & 11.60 & 9.20 & 21.21 & 122.33 & 83.21 & 21.65 & 2.00 & 35.03 \\
\hline 72 & CSR10 @ & 88.67 & 77.03 & 16.93 & 14.73 & 19.29 & 117.33 & 81.76 & 22.03 & 1.78 & 30.43 \\
\hline 73 & CST7-1 () & 106.33 & 94.30 & 15.40 & 12.17 & 22.71 & 131.00 & 79.60 & 21.30 & 4.78 & 21.05 \\
\hline 74 & CSR36 C) & 106.67 & 101.07 & 14.30 & 12.00 & 23.14 & 136.00 & 83.61 & 24.50 & 1.56 & 31.59 \\
\hline 75 & CSR27 () & 95.67 & 95.60 & 10.23 & 8.17 & 21.88 & 125.67 & 83.51 & 24.33 & 2.00 & 30.85 \\
\hline 76 & Sambha Sub1 @ & 120.67 & 77.73 & 14.13 & 11.70 & 20.03 & 122.67 & 79.94 & 21.60 & 4.78 & 25.83 \\
\hline 77 & UsarDhan 3 @) & 115.00 & 95.43 & 13.53 & 11.50 & 24.13 & 131.67 & 79.86 & 23.50 & 2.00 & 29.89 \\
\hline \multirow[t]{5}{*}{78} & PUSA44 @ & 93.67 & 79.23 & 10.27 & 8.17 & 20.10 & 115.00 & 76.51 & 19.60 & 7.00 & 18.21 \\
\hline & Mean & 99.20 & 94.12 & 12.56 & 9.74 & 21.63 & 117.44 & 77.04 & 21.77 & 3.75 & 24.73 \\
\hline & C.V. & 2.74 & 2.63 & 7.08 & 9.87 & 3.10 & 18.48 & 5.90 & 2.58 & 6.26 & 20.06 \\
\hline & C.D. $5 \%$ & 4.38 & 3.99 & 1.43 & 1.55 & 1.08 & 35.00 & 7.32 & 0.90 & 0.38 & 8.38 \\
\hline & C.D. $1 \%$ & 5.79 & 5.27 & 1.89 & 2.04 & 1.43 & 46.22 & 9.68 & 1.19 & 0.50 & 10.59 \\
\hline
\end{tabular}

Table. $2 \mathrm{~K}$ - Clustering pattern of 78 salt tolerant rice genotype

\begin{tabular}{|c|c|c|c|}
\hline Group & $\mathbf{n}$ & Within SS & Cluster Members \\
\hline 1 & 10 & 20.117 & $\begin{array}{l}\text { CARI Dhan 10, CR 2851-S-B-1-2B-1, CR 3878-245-2-4-1, CR3881-M-3-1-5-1- } \\
\text { 1-1, CSAR 1628, CSR 2016-IR18-7, IR 83421-6-B-3-1-1 CR 3364-S-2B-14-2B- } \\
\text { 1, IR84649-81-4-1-3B-CR3397-S-B-4B-1, RP 5694-36-9-5-1-1, CST7-1 @ }\end{array}$ \\
\hline 2 & 14 & 52.643 & $\begin{array}{l}\text { CR 2851-S-B-1-B-B-1, CR 3437-1*-S-200-83-1, CR 3880-10-1-9-2-2-1, CR } \\
\text { 3881-4-1-3-7-2-3, CR3884-244-8-5-6-1-1, CR3903-161-1-3-2, CSR 2016-IR18- } \\
\text { 11, CSR 2016-IR18-9, IR10206-29-2-1-1, KR 15010, KR 15016, PAU 3835-12- } \\
\text { 1-1-1, PAU 4254-14-1-2-2-2-4-1, RP 5687-420-111-5-4-2-1 }\end{array}$ \\
\hline 3 & 4 & 6.400 & CSAR 1610, CSAR1620, KS -12, Usar Dhan 3 @) \\
\hline 4 & 9 & 7.588 & $\begin{array}{l}\text { CARI Dhan 6, CSR 2016-IR18-17, CSR 2016-IR18-18, CSR 2016-IR18-8, CSR- } \\
\text { C27SM-117, NDRK 11-20, NDRK 11-22, TR 09030, CSR27 @ }\end{array}$ \\
\hline 5 & 8 & 24.328 & $\begin{array}{l}\text { CR 3883-3-1-5-2-1-2, CR 3887-15-1-2-1, CR 3890-35-1-3-4, CSA 2016-IR18-6, } \\
\text { CSR 2016-IR18-10, CSR RIL-01-IR165,CSR-2748-197, PAU 7114-3480-1-1-1- } \\
0\end{array}$ \\
\hline 6 & 4 & 40.597 & $\begin{array}{l}\text { CARI Dhan 11, CSR-2748-4441-193, CSRC(S)47-7-B-B-1-1, IR52280-117-1-1- } \\
3\end{array}$ \\
\hline 7 & 5 & 0.807 & CSR 2016-IR18-12, KR15006, NDRK 11-21, NDRK 11-24, CSR36 @ \\
\hline 8 & 12 & 30.198 & $\begin{array}{l}\text { CR 2851-S-1-6-B-B-4, CR 3884-244-8-5-11-1-1, CR 3904-162-1-5-1, CSR } \\
\text { 2016-IR18-1, CSR 2016-IR18-14, PAU 5563-23-1-1, RP 5440-302-100-7-6-3-2, } \\
\text { RP-320-4-3-2-1, Sambha Sub1, TR 09027, Sambha Sub1 @, PUSA44 @ }\end{array}$ \\
\hline 9 & 12 & 19.108 & $\begin{array}{l}\text { CR3882-7-1-6-2-2-1, CSAR 1604, SR 2016-IR18-15, CSR 2016-IR18-16, CSR } \\
\text { 2016-IR18-2, CSR 2016-IR18-3, CSR 2016-IR18-5, CSR-2748-4441-195, PAU } \\
\text { 3835-36-6-3-3-4, RAU 1397-14, RP-5683-101-85-30-2-3-1, CSR10 @ }\end{array}$ \\
\hline
\end{tabular}


Table.3 K- Cluster mean for 9 clusters in salt tolerant rice genotypes

\begin{tabular}{|c|c|c|c|c|c|c|c|c|c|c|}
\hline Cluster & $\begin{array}{c}\text { Days to } \\
50 \% \\
\text { Flowering }\end{array}$ & $\begin{array}{c}\text { Plant } \\
\text { Height } \\
\text { (cm) }\end{array}$ & $\begin{array}{l}\text { Tillers } \\
\text { Plant }^{-1}\end{array}$ & $\begin{array}{c}\text { Producti } \\
\text { ve Tillers } \\
\text { Plant }^{-1}\end{array}$ & $\begin{array}{c}\text { Panicle } \\
\text { Length } \\
\text { (cm) }\end{array}$ & $\begin{array}{c}\text { Filled } \\
\text { Grains } \\
\text { Panicle }^{-1}\end{array}$ & $\begin{array}{c}\text { Spikelet } \\
\text { Fertility } \\
(\%)\end{array}$ & $\begin{array}{c}1000 \text { Seed } \\
\text { Weight } \\
\text { (g) }\end{array}$ & $\begin{array}{c}\text { Stress } \\
\text { at } \\
\text { reprodu } \\
\text { ctive } \\
\text { stage }\end{array}$ & $\begin{array}{l}\text { Grain } \\
\text { Yield } \\
\text { (gm/ } \\
\text { plant) }\end{array}$ \\
\hline 1 Cluster & 98.000 & 96.864 & 12.480 & 9.607 & 21.027 & 115.967 & 76.323 & 21.841 & 3.678 & 24.59 \\
\hline 2 Cluster & 104.024 & 94.520 & 12.802 & 9.314 & 20.771 & 119.738 & 76.918 & 20.102 & 5.810 & 17.50 \\
\hline 3 Cluster & 96.750 & 84.725 & 13.333 & 10.967 & 21.833 & 127.250 & 79.514 & 23.944 & 1.750 & 33.66 \\
\hline 4 Cluster & 95.926 & 98.778 & 12.422 & 10.019 & 21.630 & 118.852 & 77.619 & 23.755 & 2.333 & 29.50 \\
\hline 5 Cluster & 93.333 & 111.592 & 12.650 & 10.017 & 21.483 & 124.750 & 78.597 & 21.633 & 1.875 & 32.11 \\
\hline 6 Cluster & 85.667 & 136.308 & 12.325 & 9.125 & 23.169 & 118.000 & 78.675 & 21.455 & 1.750 & 34.21 \\
\hline 7 Cluster & 90.267 & 95.807 & 13.047 & 10.373 & 24.035 & 119.467 & 78.260 & 25.353 & 2.511 & 30.76 \\
\hline 8 Cluster & 109.278 & 75.996 & 11.914 & 9.300 & 20.949 & 113.250 & 75.860 & 20.238 & 6.315 & 15.94 \\
\hline 9 Cluster & 99.889 & 82.684 & 12.661 & 9.944 & 22.358 & 109.944 & 75.628 & 21.693 & 3.009 & 24.94 \\
\hline
\end{tabular}

Table.4 Cluster Member: Ward of salt tolerant genotypes

\begin{tabular}{|c|c|c|}
\hline Cluster & Number & Cluster Members \\
\hline 1 & 14 & $\begin{array}{l}\text { CARI Dhan 10,CR 3880-10-1-9-2-2-1,CR3882-7-1-6-2-2-1,CSR-2748- } \\
\text { 4441-195,RAU 1397-14,CSR10 C,CSAR 1604,RP-5683-101-85-30-2-3- } \\
\text { 1,CSR 2016-IR18-3,CSAR 1610,CSR-C27SM-117,CSAR1620,TR } \\
\text { 09030,KS -12 }\end{array}$ \\
\hline 2 & 4 & $\begin{array}{l}\text { CSR 2016-IR18-15,CSR 2016-IR18-16,CSR 2016-IR18-5,CSR 2016- } \\
\text { IR18-2 }\end{array}$ \\
\hline 3 & 22 & $\begin{array}{l}\text { CARI Dhan 6,CSR RIL-01-IR165,CSR 2016-IR18-8,CSR27 @,CSR36 } \\
\text { ○,Usar Dhan } 3 \text { @,CR 3883-3-1-5-2-1-2,IR84649-81-4-1-3B-CR3397-S- } \\
\text { B-4B-1,CR 2851-S-B-1-2B-1,IR 83421-6-B-3-1-1 CR 3364-S-2B-14- } \\
\text { 2B-1,CSR 2016-IR18-17,NDRK 11-22,CR 3878-245-2-4-1,CR3881-M- } \\
\text { 3-1-5-1-1-1,PAU 7114-3480-1-1-1-0,CSR-2748-197 } \\
\text { NDRK 11-20,CSR 2016-IR18-12,CSR 2016-IR18-18,NDRK } \\
\text { 21,NDRK 11-24,KR15006 }\end{array}$ \\
\hline 4 & 2 & CARI Dhan 11,CSRC(S)47-7-B-B-1-1 \\
\hline 5 & 6 & $\begin{array}{l}\text { CR 3890-35-1-3-4,IR52280-117-1-1-3,CSR-2748-4441-193,CSA 2016- } \\
\text { IR18-6,CSR 2016-IR18-10,CR 3887-15-1-2-1 }\end{array}$ \\
\hline 6 & 6 & $\begin{array}{l}\text { CR 2851-S-1-6-B-B-4,CSR 2016-IR18-14,RP-320-4-3-2-1,PAU 5563- } \\
\text { 23-1-1,RP 5440-302-100-7-6-3-2,Sambha Sub1 C) }\end{array}$ \\
\hline 7 & 9 & $\begin{array}{l}\text { CR 3437-1*-S-200-83-1,PAU 3835-36-6-3-3-4,CSR 2016-IR18-9,KR } \\
\text { 15016,TR 09027,CSR 2016-IR18-1,PUSA44 C) } \\
\text { CR 3884-244-8-5-11-1-1,KR 15010 }\end{array}$ \\
\hline 8 & 8 & $\begin{array}{l}\text { CR 2851-S-B-1-B-B-1,CR3903-161-1-3-2,CR 3904-162-1-5-1,Sambha } \\
\text { Sub1,CR 3881-4-1-3-7-2-3,RP 5687-420-111-5-4-2-1,IR10206-29-2-1-1, } \\
\text { CR3884-244-8-5-6-1-1 }\end{array}$ \\
\hline 9 & 7 & $\begin{array}{l}\text { CSAR 1628, CSR 2016-IR18-7,CSR 2016-IR18-11,PAU 4254-14-1-2-2- } \\
\text { 2-4-1,RP 5694-36-9-5-1-1,PAU 3835-12-1-1-1,CST7-1 () }\end{array}$ \\
\hline
\end{tabular}


Table.5 Euclidean²: Cluster Distances: Ward of salt tolerant genotypes

\begin{tabular}{|c|c|c|c|c|c|c|c|c|c|}
\hline & $\begin{array}{c}1 \\
\text { Cluster }\end{array}$ & $\begin{array}{c}2 \\
\text { Cluster }\end{array}$ & $\begin{array}{c}3 \\
\text { Cluster }\end{array}$ & $\begin{array}{c}4 \\
\text { Cluster }\end{array}$ & $\begin{array}{c}5 \\
\text { Cluster }\end{array}$ & $\begin{array}{c}6 \\
\text { Cluster }\end{array}$ & $\begin{array}{c}7 \\
\text { Cluster }\end{array}$ & $\begin{array}{c}8 \\
\text { Cluster }\end{array}$ & $\begin{array}{c}9 \\
\text { Cluster }\end{array}$ \\
\hline 1 Cluster & 116.673 & 172.029 & 183.177 & 968.354 & 409.288 & 422.851 & 400.443 & 579.947 & 279.600 \\
\hline 2 Cluster & & 51.753 & 168.866 & 945.709 & 419.343 & 295.620 & 337.771 & 371.517 & 164.089 \\
\hline 3 Cluster & & & 107.323 & 608.137 & 216.157 & 572.199 & 477.321 & 512.713 & 215.185 \\
\hline 4 Cluster & & & & 178.996 & 279.854 & 1649.706 & 1332.879 & 1126.068 & 773.294 \\
\hline 5 Cluster & & & & & 133.85 & 1025.527 & 851.416 & 798.784 & 425.782 \\
\hline 6 Cluster & & & & & & 101.840 & 216.753 & 278.966 & 275.870 \\
\hline 7 Cluster & & & & & & & 152.255 & 300.357 & 245.122 \\
\hline 8 Cluster & & & & & & & & 125.463 & 160.785 \\
\hline 9 Cluster & & & & & & & & & 64.182 \\
\hline
\end{tabular}

Table.6 Cluster Mean of 10 traits for salt tolerant genotypes

\begin{tabular}{|c|c|c|c|c|c|c|c|c|c|c|}
\hline & $\begin{array}{c}\text { Days to } 50 \% \\
\text { flowering }\end{array}$ & $\begin{array}{l}\text { Plant height } \\
\text { (cm) }\end{array}$ & $\begin{array}{l}\text { Tillers/ } \\
\text { Plant }\end{array}$ & $\begin{array}{c}\text { Productive } \\
\text { Tillers/ } \\
\text { plant }\end{array}$ & $\begin{array}{c}\text { Panicle } \\
\text { Length } \\
\text { (cm) }\end{array}$ & $\begin{array}{c}\text { Filled grains/ } \\
\text { panicle }\end{array}$ & $\begin{array}{c}\text { Spikelet } \\
\text { Fertility } \\
(\%)\end{array}$ & $\begin{array}{c}\text { Test } \\
\text { Weight }\end{array}$ & $\begin{array}{c}\text { Stress score } \\
\text { at reproductive } \\
\text { stage }\end{array}$ & $\begin{array}{l}\text { Grain } \\
\text { Yield } \\
\text { g/plant }\end{array}$ \\
\hline 1 Cluster & 94.476 & 81.929 & 12.436 & 9.852 & 20.385 & 121.881 & 78.276 & 22.147 & 2.270 & 2987.818 \\
\hline 2 Cluster & 109.333 & 89.302 & 12.958 & 9.325 & 24.908 & 92.833 & 71.462 & 21.546 & 3.333 & 2302.250 \\
\hline 3 Cluster & 94.818 & 99.394 & 12.527 & 9.932 & 22.339 & 119.955 & 77.437 & 23.424 & 2.525 & 2874.303 \\
\hline 4 Cluster & 86.167 & 145.850 & 13.500 & 10.000 & 21.975 & 118.667 & 79.945 & 19.883 & 2.000 & 3349.333 \\
\hline 5 Cluster & 91.944 & 119.650 & 12.500 & 9.861 & 22.219 & 125.611 & 79.046 & 22.017 & 1.500 & 3516.889 \\
\hline 6 Cluster & 114.889 & 73.481 & 12.211 & 9.472 & 21.083 & 118.389 & 76.436 & 19.267 & 5.907 & 1728.593 \\
\hline 7 Cluster & 89.741 & 76.826 & 11.674 & 9.126 & 21.298 & 115.333 & 76.187 & 19.986 & 5.926 & 1650.852 \\
\hline 8 Cluster & 115.917 & 101.210 & 12.617 & 9.179 & 20.905 & 115.792 & 75.867 & 20.852 & 6.917 & 1520.167 \\
\hline 9 Cluster & 106.143 & 99.789 & 13.857 & 10.662 & 21.186 & 111.143 & 76.945 & 21.789 & 4.968 & 1926.952 \\
\hline
\end{tabular}

Fig.1

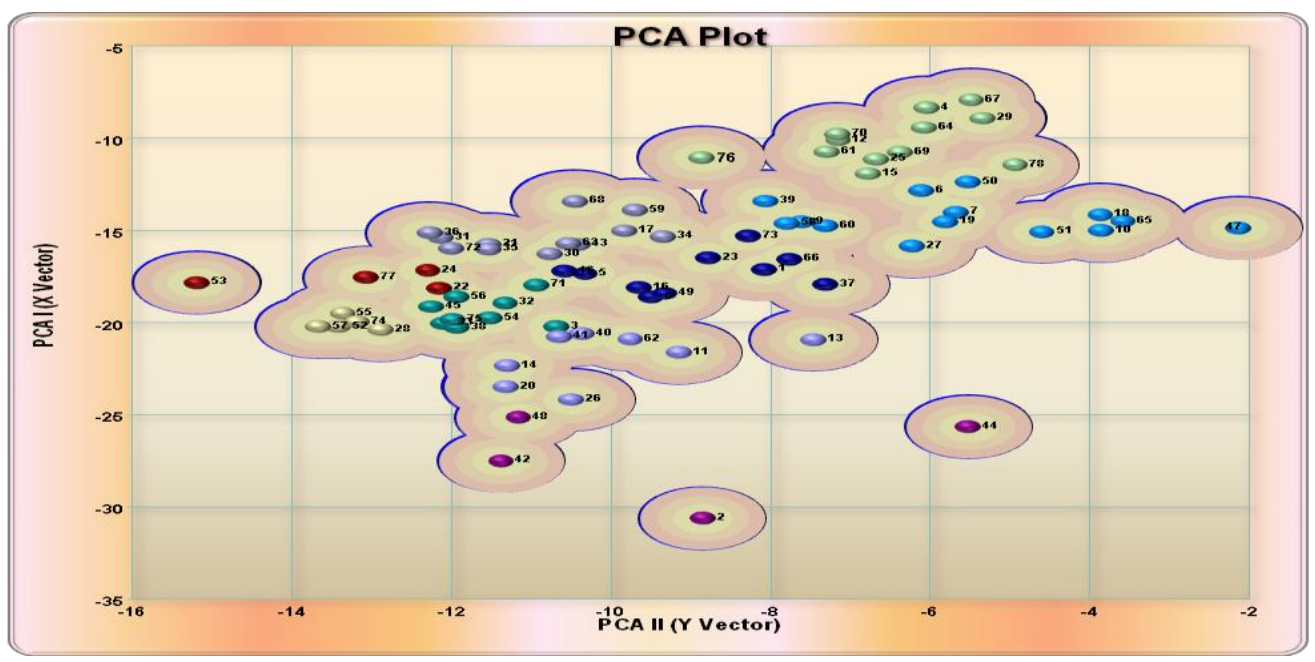

- Cluster $1 \bullet$ cluster $2 \bullet$ cluster $3 \bullet$ cluster4 $\bullet$ cluster $5 \bullet$ cluster6 $\bullet$ cluster $7 \bullet$ cluster $8 \bullet$ cluster 9 
Int.J.Curr.Microbiol.App.Sci (2020) 9(11): 359-367

Fig.2

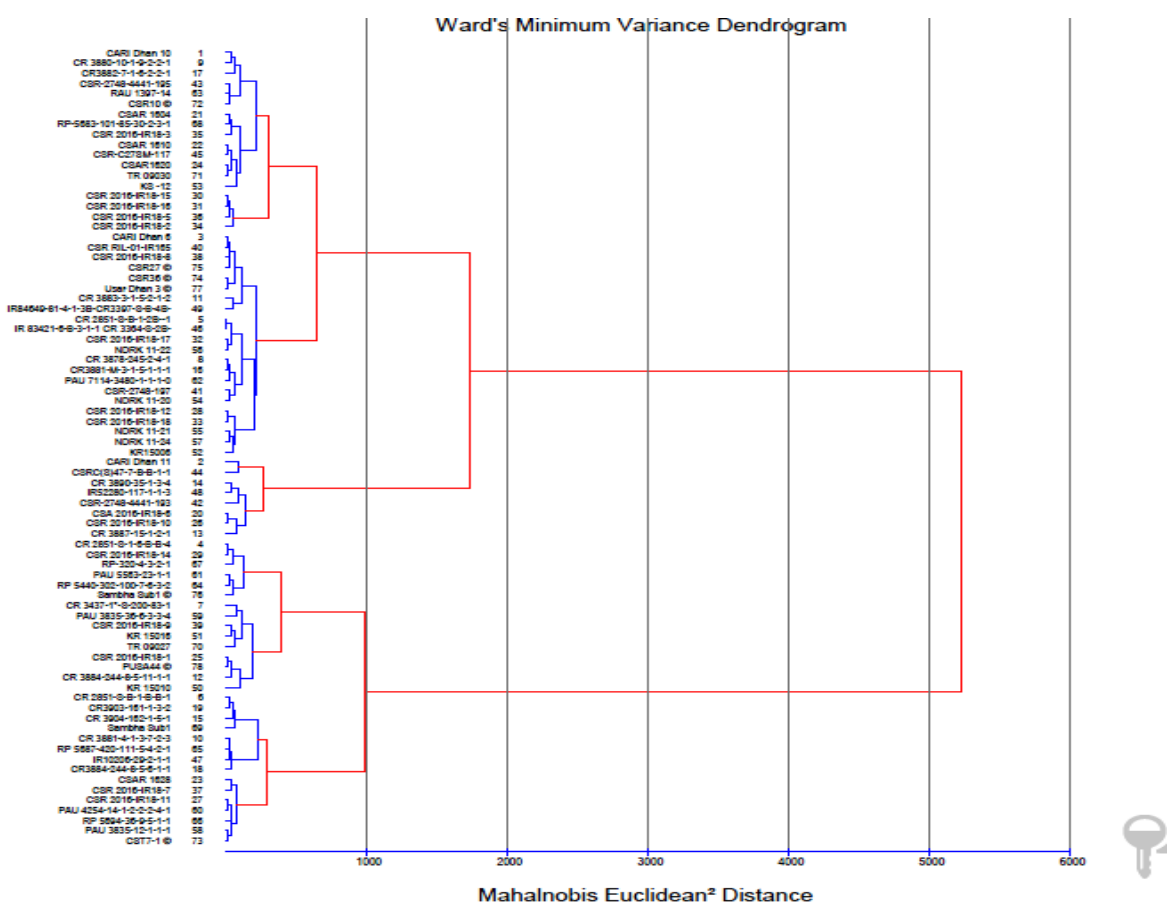

Fig.3

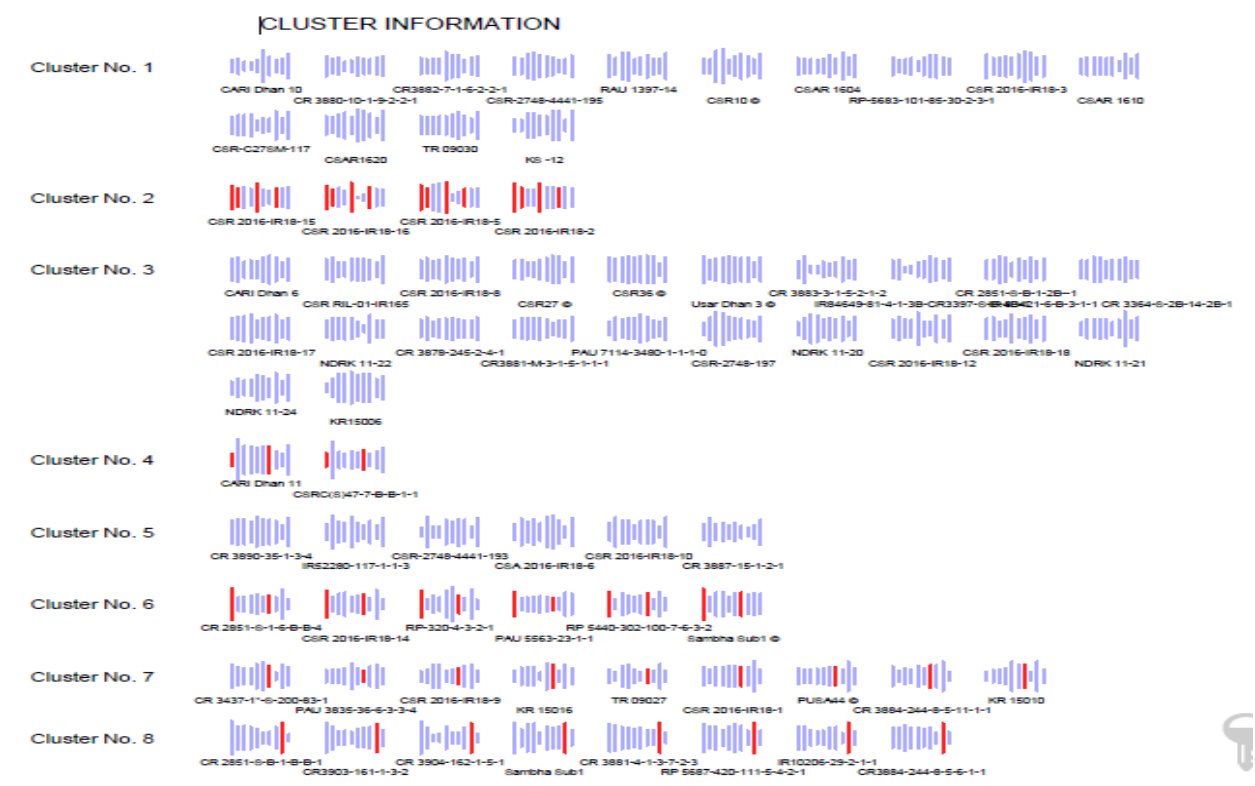

Cluster No. 9

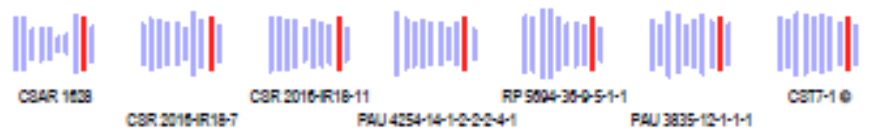


On the basis of difference within SS, seventy eight genotypes were grouped into nine clusters in the present study by both k-Means Clustering, and Euclidian revealed the genotypes of heterogeneous origin were frequently present in same cluster (Groenendyk et al., 2014).

Although the genotypes originated in same place or geographic region were also found to be grouped together in same cluster, the instances of grouping of genotypes of different origin or geographical regions in same cluster were observed in case of all the clusters. k-Means Clustering showed that Cluster II, VIII, IX, I, IV, V consisted of 14, 12, 12, 10, 9 and 8 entries and Cluster III, IV and VII contains 4,4 and 5 genotypes, respectively, while in Euclidian, cluster V, III,I,IV,II comprised 23,19,15,9 and 7 entries, respectively. Although, cluster IV have equal numbers of entries but all the genotypes were different.

The average maximum inter cluster difference within SS values was observed between cluster II\&VII followed by cluster II\&III, cluster II \&IV, cluster VI \&VII, and cluster III \& VI indicated great extent of diversity between these groups (Table 2 and 3). Cluster differences observed highest between cluster IV and six followed by cluster $\mathrm{V}$ and six. Therefore, it is suggested that any superior genotypes of cluster II and VI may be crossed with any superior genotype of cluster VII and III to produce desirable recombinants in hybridization programme and also revealed that the genotypes present in a cluster have little genetic divergence from each other with respect to aggregate effect of ten characters under study, while much more genetic diversity was observed between the genotypes belonging to different clusters Ranjbar et al., (2007); Sapra and Lal (2003); Maqbool et al., (2010) and Ahmadizadeh et al., (2011).
A comparison of cluster mean for the studied characters indicated significant divergence between the groups. Some groups showed highest and other showed lowest value for the different characters in respect of the traits as fall in to different clusters in both types of cluster analysis. Low conformity was observed in placing of genotypes in both clustering techniques but it was provided important information on some genotypes which have common placing in both clustering pattern.

In cluster I genotype CARI dhan 10, cluster third Usar dhan 3 and cluster five CR 389035-1-3-4, CSA -2016 and CSR -2016-IR-1810 are placed as common genotypes by both clustering pattern.

In merit of mean yield performance, CSR 2016-IR-18-10 placed as highest second yielder followed by CSA -2016, CARI dhan 10 , Usar Dhan 3 possessed $4^{\text {th }}, 16^{\text {th }}$ and $25^{\text {th }}$ rank. These genotypes were considered more stable across the environment (Table 1, 2 and 4).

In conclusion, it is clearly reflected wide variation from one cluster to another in respect of cluster means for ten characters, which indicated that genotypes having distinctly different mean performance for various characters were separated into different clusters (Table 5 and 6). Both clustering techniques have different results in placing of genotypes in respective cluster but it was provided important information on some genotypes which have common placing in both clustering pattern. The crossing between the entries belongings to cluster pairs having large difference within sum of square and possessing high cluster means for one or other characters to be improved may be recommended for isolating desirable salt tolerant rice lines. 


\section{References}

Ahmadizadeh, M., Valizadeh, M., Shahbazi, H., Zaefizadeh, M. and Habibpor, M. 2011. Morphological diversity and interrelationships traits in durum wheat landraces under normal irrigation and drought stress conditions. Adv. Environ. Biol., 5(7): 1934-1940.

Derek Groenendyk Kelly Thorp Ty Ferre Wade Crow Doug Hunsaker 2014.A KMeans Clustering approaches To assess Wheat Yield Prediction Uncertainty with a HYDRUS -1D coupled crop model. international Environmental Modeling and Software Society.

Manju Kaushik and Bhawana mathur 2014; comparative of K-Means and Hierarchical Clustering Techniques. International journal of software \& hardware research in Engineering. Vol.2 Issue 6.

Escobar-Hernandez, A., 2005;Troyo-dieguez, E., Garcia-hernandezcontreras, J.L., Murillo-amador, B. and Lopez-aguilar, R. Principal component analysis to determine forage potential of salt grass Distichlis spicata L. (Grrene) in coastal ecosystems of Baja Califoniasur, Mexico. Tech. Pecu. Mex.), 43: 13-25.

Escobar-Hernandez, A., Troyo-dieguez, E., Garcia-hernandezcontreras, J.L., Murillo-amador, B. and Lopez-aguilar, R. 2005. Principal component analysis to determine forage potential of salt grass Distichlis spicata L. (Grrene) in coastal ecosystems of Baja Califoniasur, Mexico. Tech. Pecu. Mex., 43: 13-25.
Hartigan, J., and Wang, M. 1979. A K-means clustering algorithm. Applied Statistics, 28, 100-108.

Kromdijk J, Long S P. 2016. One crop breeding cycle from starvation? How engineering crop photosynthesis for rising $\mathrm{CO} 2$ and temperature could be one important route to alleviation. Proc Royal Soc B: Biol Sci, 283: 20152578

Lloyd, S. 1957. Least squares quantization in pcm. Bell Telephone Laboratories Paper, Marray Hill.

MacQueen, J. 1967. Some methods for classification and analysis of multivariate observations. Proc. 5th Berkeley Symposium, 281-297.

Mahalanobis, P C. 1930; On Test and Measures of groups divergence Part I. Theoretical Formulae J.Asiatic Sco. Bengal 26, 541-586.

Maqbool, R., Sajjad, M. and Khaliq, I. 2010. Morphological diversity and traits association in bread wheat (Triticum aestivum L.). American-Eur. J. Agric. Environ. Sci., 8(2): 216- 224.

R. Shivramakrishnan, R. Vinoth, Ajay Arora, G.P. Singh, B. Kumar and V.P. Singh, 2016 Characterization of wheat genotypes for stay green and physiological traits by principal component analysis under drought condition; International Journal of Agricultural Sciences, 12 (2) :.245-251

Sapra, R.L. and Lal, S.K. 2003. A strategy for selecting diverse accessions using principal component analysis from a large germplasm collection of soybean. Pl. Genetic. Resour., 1: 151-156.

\section{How to cite this article:}

Ashutosh Kashyap, Vijay Kumar Yadav, Poonam Singh, P. K. Singh and Shweta. 2020. KMean and Euclidian Cluster Analysis for Salt Tolerance Rice Genotypes under Alkaline Soil Condition. Int.J.Curr.Microbiol.App.Sci. 9(11): 359-367.

doi: https://doi.org/10.20546/ijcmas.2020.911.043 